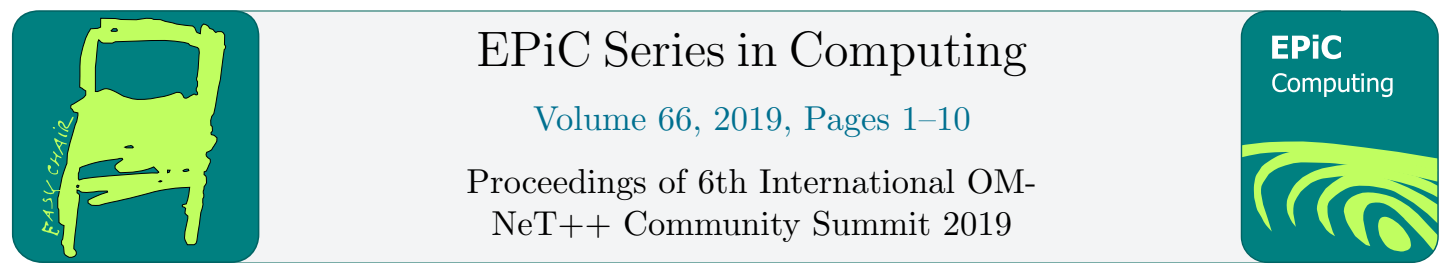

\title{
Evaluating Modern Data Centre Transport Protocols in OMNeT++/INET
}

\author{
Mohammed Alasmar and George Parisis \\ School of Engineering and Informatics \\ University of Sussex, UK \\ M.Alasmar@sussex.ac.uk, G.Parisis@sussex.ac.uk
}

\begin{abstract}
In this paper we present our work towards an evaluation platform for data centre transport protocols. We developed a simulation model for $\mathrm{NDP}^{1}$, a modern data transport protocol in data centres, a FatTree network topology and per-packet ECMP load balancing. We also developed a data centre environment that can be used to evaluate and compare data transport protocols, usch as NDP and TCP. We describe how we integrated our model with the INET Framework and present example simulations to showcase the workings of the developed framework. For that, we ran a comprehensive set of experiments and studied different components and parameters of the developed models.
\end{abstract}

\section{Introduction}

The study of network protocols for Data Centre Networks (DCNs) has become increasingly important, given that data centres support all major Internet services, such as search (e.g. Google), social networking (e.g. Facebook), cloud services (e.g. Amazon EC2) and video streaming (e.g. NetFlix). DCNs consist of a large number of commodity servers and switches and support multiple paths among servers. Recent research on data centre networking is based on various simulation tools and respective models for network protocols [16, 20, 19].

$\mathrm{OMNeT}++[26]$ is an excellent candidate for developing models for data centre networks and respective protocols, and more work is required for establishing it as the de facto simulator for this research community. This is possible through the INET Framework, which is built on top of the simulation core provided by OMNeT ++ . Omnet++ and INET is built around the concept of modules that communicate by message passing. Protocols are represented by components, which can be combined to form hosts, routers, switches and other networking devices. What makes this framework ideal for evaluating DCN protocols is that new modules can be easily integrated with the existing modules. DCN topologies (e.g. FatTree [1]) can be easily built and parameterised using Omnet++ NED language.

Modelling DCN protocols in OMNeT++. Recently, some DCN-related research has been based on OMNeT++/INET [21, 5, 11, 3, 4]. Achieving the critical mass of researchers that use $\mathrm{OMNeT}++$ for evaluating data centre networks and protocols requires making more modern

${ }^{1}$ https://github.com/mohammedalasmar/ndpTcpDatacentreOmnetppModel (OMNeT++-5.2.1 \& INET-3.6.3) 
protocols available in the $\mathrm{OMNeT}++$ environment. Large-scale simulations are crucial for the DCN research community given that access to real-world deployments is very difficult. Developing models for DCNs in OMNeT++ would also ensure reproducibility, revisability (dynamic debugging and profiling) and control over the studied traffic workloads (generating realistic traffic workloads in a deterministic fashion) [25].

Efficient data centre transport protocols. In DCNs, an efficient data transport mechanism is crucial to provide near-optimal completion times for short transfers and high goodput for long flows. The performance of TCP in DCNs is problematic due to TCP Incast [10], queue build-up and buffer pressure [14, 24, 17] and per-flow ECMP collisions. TCP performance can get singificantly degraded because of frequent retransmissions of lost packets $[6,17]$. Recently, a large body of work aimed at tackling various aspects of data centre transport: proposed approaches usually focus on either achieving low latency $[6,22,27,21,7]$ or high throughput $[17,23,12,2]$. NDP [14] appears to perform well with respect to both low latency and high throughput requirements by combining a number of data transport mechanisms.

NDP and FatTree models. In this paper we present an OMNeT++/INET framework for evaluating data transport protocols (NDP and TCP) in data centres. This includes: (1) a model for building FatTree topologies to evaluate the performance of TCP, NDP and other community-developed protocol models for data centres (\$2), (2) a model for per-packet and perflow Equal-Cost Multi-Path (ECMP) load balancing in a FatTree topology (§3), (3) a model for NDP ( $\$ 4$ and $\S 5)$, and (4) a central traffic scheduler for scheduling flows in the simulated network and setting up simulation parameters for experimenting with the above-mentioned contributions $(\S 5)$.

\section{FatTree Topology}

Among the recently proposed DCN topologies, FatTree, which originated from the Clos switching network, is widely used [1]. We developed a FatTree topology generator using the NED language. FatTree data centres allow any two servers to communicate by fully utilising network resources and ensuring non-blocking behaviour. The role of core switches is to forward traffic among aggregation switches, and that of the aggregation switch is to inter-connect core and edge switches. The edge switches reside at lowest level of the topology, and forward traffic between hosts and aggregation switches (see Figures 1a\&2). The size of a FatTree topology depends on the number of pods it consists of $(k)$. A FatTree network consists of three layers: the core layer, aggregation layer and edge layer. In a $k$-ary FatTree topology there are $k^{2} / 4$ core switches which is the same number as the shortest-paths between any two servers that are connected to any pod in the network. Each pod contains $k$ servers and $k$ switches. These switches are divided into two layers each consisting of $k / 2$ switches. The first layer is the edge where each switch is connected to $k / 2$ of the servers (a rack) in the same pod, while the second layer is the aggregation layer where each switch is connected to $k / 2$ of the core switches. Each core switch is connected to one aggregation switch of each pod. The maximum number of servers in a FatTree with $k$ pods is $k^{3} / 4$. All switches have the same number of ports which is equal to $k$. Table 1 summarises the construction of a $k$-ary FatTree (with examples when $k=$ Generating . FatTree networks. The implementation of the FatTree topology using the NED language is based on the values in Table 1. The only required input value to generate the topology is $k$. Figure 1 shows an example of a generated FatTree topology when $k=4$. The FatTree module is a network (complex) module that contains two simple modules: Pod and Rack submodules. The NED code for the FatTree module is depicted in Figure 1a, which is used to create a single link between each core switch and each pod. 


\begin{tabular}{|l|l|l|l|l|}
\hline Pods & $k$ & 4 & 8 & 10 \\
\hline Servers & $k \times k / 2 \times k / 2$ & 16 & 128 & 250 \\
\hline Core switches (= servers in each Pod) & $k / 2 \times k / 2$ & 4 & 16 & 25 \\
\hline Edge switches in each Pod (racks) & $k / 2$ & 2 & 4 & 5 \\
\hline Aggregation switches in each Pod & $k / 2$ & 2 & 4 & 5 \\
\hline Total edge/aggregation switches & $k \times k / 2$ & 8 & 24 & 50 \\
\hline Switch ports & $k$ & 4 & 8 & 10 \\
\hline $\begin{array}{l}\text { Equal-cost path between any pair of } \\
\text { servers (at different pod) }\end{array}$ & $k / 2 \times k / 2$ & 4 & 16 & 50 \\
\hline
\end{tabular}

Table 1: $k$-ary FatTree topology architecture: examples when $k=4,8$ and 10

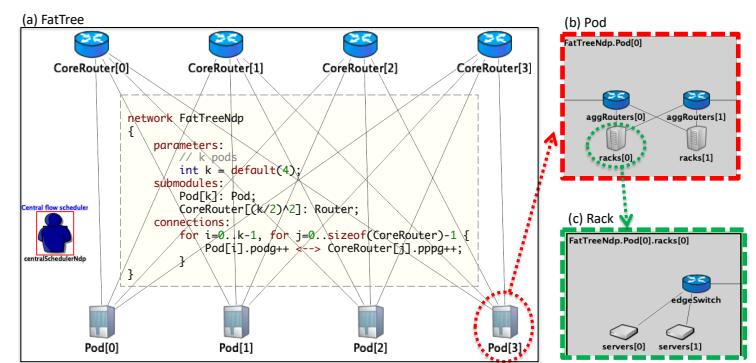

(a) FatTree topology $(k=4)$

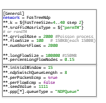

(b) centralScheduler ini file

Figure 1: FatTree implementation in NED language including a central scheduler node

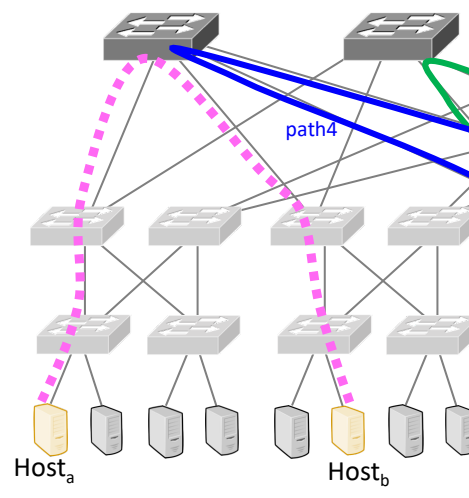

(a) Per-flow ECMP $= \pm n=n$

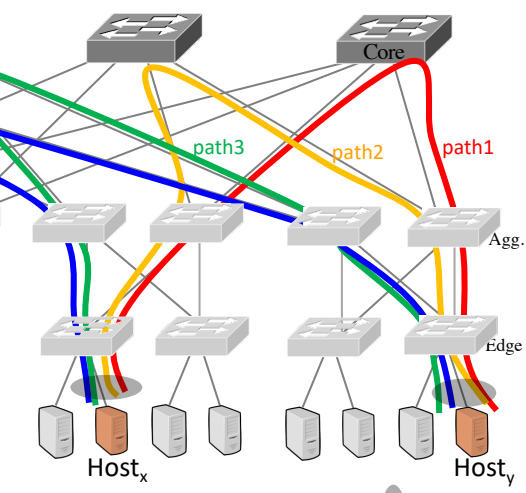

(b) Per-packet ECMP

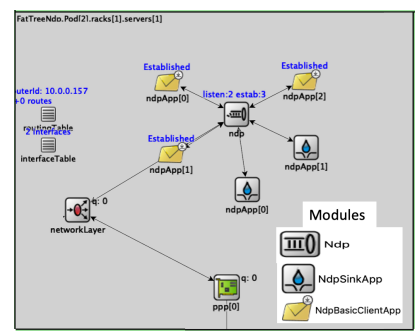

Figure 2: (a) Per-flow ECMP vs (b) per-packet ECMP in 4-FatTree

\section{Per-packet and per-flow ECMP}

Modern data centre transport protocols exploit the existence of multiple equal-cost paths in FatTree networks to better balance traffic in the network, eliminate hotspots and achieve high throughput. ECMP is used for packet forwarding in the network [15]. In per-flow ECMP, packets are classified into different flows by hashing the each packet's 5-tuple (source IP address, destination IP address, protocol number, source port number and destination port number). Packets of the same flow go over the same link, as depicted in Figure 2a. Per-flow hashing ensures that packets belonging to the same flow (or sub-flow in MultiPath TCP) will arrive in 
order to their destination. However, this can cause significant underutilisation in the network due to collisions of large flows (i.e. when a large number of flows cross the same link while other links are not used) and this can significantly reduce throughput, as discussed in [13]. In addition, per-flow load-balancing can result in unequal link utilisation and hotspots. In per-packet ECMP, packet forwarding is randomised over all equal-cost links used for load balancing, as shown in Figure 2b. Per-packet multipath forwarding is a good option when using a data transport protocol that can tolerate reordering (e.g., NDP [14][3]). As per-packet ECMP may result in packets arriving out of order, it cannot be used with data transport protocols that are sensitive to packet reordering (e.g., TCP).

A model of per-packet and per-flow ECMP in INET. We implemented both per-packet and per-flow ECMP by updating the source code that is provided in INETv3.6.3 network layer. Routing in INET is done in five main steps as follows: (1) building topology and assigning addresses (NetworkConfiguratorBase::Topology), (2) setting links and node weights (IPv4NetworkConfigurator::computeConfiguration), (3) using Dijkstra's Algorithm for multiple paths (Topology::calculateWeightedSingleShortestPathsTo), (4) adding a route to all destinations in the network (IPv4NetworkConfigurator::addStaticRoutes) and (5) generating the routing tables (IPv4RoutingTable::printRoutingTable).

When implementing ECMP, we updated step 3 so that all shortest paths to all destinations are registered. Additionally, we updated step 4 to include the updates in step 3 when adding routes to all destinations. The routing tables in step 5 are automatically updated. Finally, we implemented the hashing function in IPv4RoutingTable::findBestMatchingRouteEcmp (which is called by IPv4::routeUnicastPacket). There are two options for hashing:

- per-packet ECMP: selectPath = rand()\% numPossibleEcmpRouts, and

- per-flow ECMP: selectPath = hashValue\% numPossibleEcmpRouts, where the hashValue is calculated based on the 5-tuple (we also included the router's name in this hashing).

\section{A model of NDP in INET}

NDP aims at offering both low latency and high throughput in FatTree data centre networks. NDP combines several ideas into a clean slate protocol design. NDP exhibits very good performance by employing receiver-driven flow control and packet trimming. NDP will be deployable when P4 switches [9] are deployed in data centres. NDP operation can be summarised as follows (and depicted through the numbered circles in Figure 4). Senders are allowed to send an initial window of data at line rate (circles 1 and 2). Switches use shallow buffers (e.g. 8 packets long) with two queues: the data (used for data packets only) and control (high priority) queue (for PULL, ACK, NACK and Header packets). If the data queue overflows, the packet payload is trimmed and the header is priority-forwarded (circles 3, 4, 5 and 6). At the receiver, an ACK for each data packet received and a NACK for each header will be sent immediately to the sender (circles 7 and 8 ). The receiver has a shared PULL queue between all active connections. The receiver adds a PULL packet for every received header or data packet (circle 9). The receiver paces PULL packets so as to fill the receiver's incoming link. Pacing is across all connections, so that the aggregate rate matches the receiver's link speed (circle 10). The goal is to keep the incoming link full, so the receiver spaces pull requests accordingly (e.g. assuming each incoming packet has the same MTU size $=1500 \mathrm{~B}$, then the receiver sends a packet every $\frac{M T U}{1 \text { Gbps }}=12 \mu$

${ }^{2}$ This is an abstract diagram of NDP functionality - the details of NDP can be found in [14] 


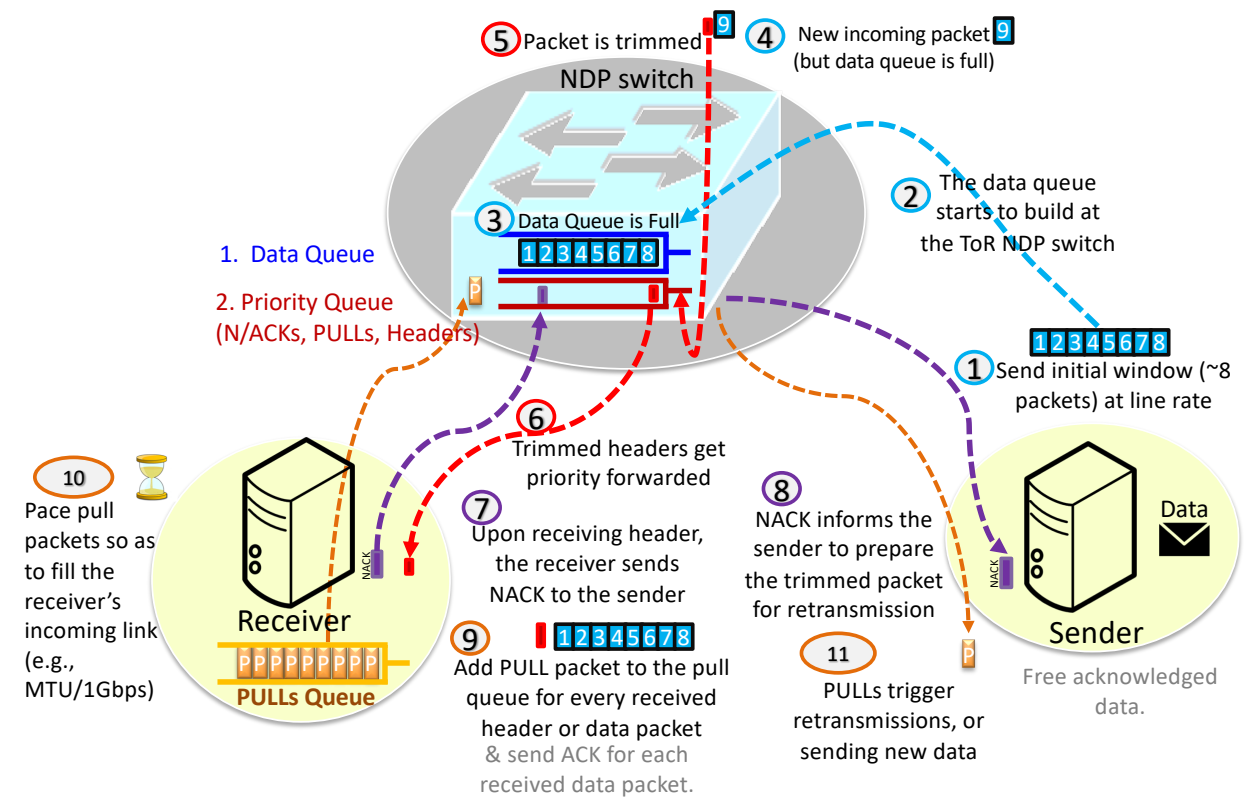

Figure 4: NDP operation ${ }^{2}$

seconds if the receiver's link speed is 1Gbps). At the sender, PULL requests trigger either a retransmission or a new data packet (circle 11).

Here we describe how we implemented NDP in INET. Our implementation follows the TCP model in INET. We developed StandardHostNdp, a predefined NED type which is an OM$\mathrm{NeT}++$ compound module that is composed of the following components:

Applications. There are two main applications that can communicate with the NDP layer, as shown in Figure 3. The first application is the NdpBasicClientApp module, which is used by NDP senders to start a connection. The second application module is NdpSinkApp which is used by NDP receivers to listen for incoming connections. NDP applications and the NDP layer communicate with each other by sending $c$ Message objects. These messages are specified in the NDPCommand.msg file. The NDPCommandCode enumeration defines the types of messages that are sent by the application to the NDP layer. These are the main message types: $N D P_{-} C_{-} O P E N_{-} A C T I V E$ : active open, NDP_C_OPEN_PASSIVE: passive open, NDP_C_SEND: send data and $N D P_{-} C_{-} C L O S E$ : no more data to send. Each command message should have attached control information of type NDPCommand. For example, the command message NDP_C_OPEN_ACTIVE requires this control information to be attached: connId, localAddr, locarPrt, remoteAddr, remotePrt and numPacketsToSend. In Figure 5, NdpSinkApp can open a local port by sending a NDP_C_PASSIVE_OPEN to the NDP layer with control information that contains the local address and port. NdpBasicClientApp opens a connection to a remote server by sending a NDP_C_OPEN_ACTIVE command to the NDP layer.

NDP layer.The NDP module creates an NDPConnection object upon receiving either an active or passive open command from an NDP application. The main message kinds that the application receives from the NDP layer are: NDP_I_ESTABLISHED: connection established, 


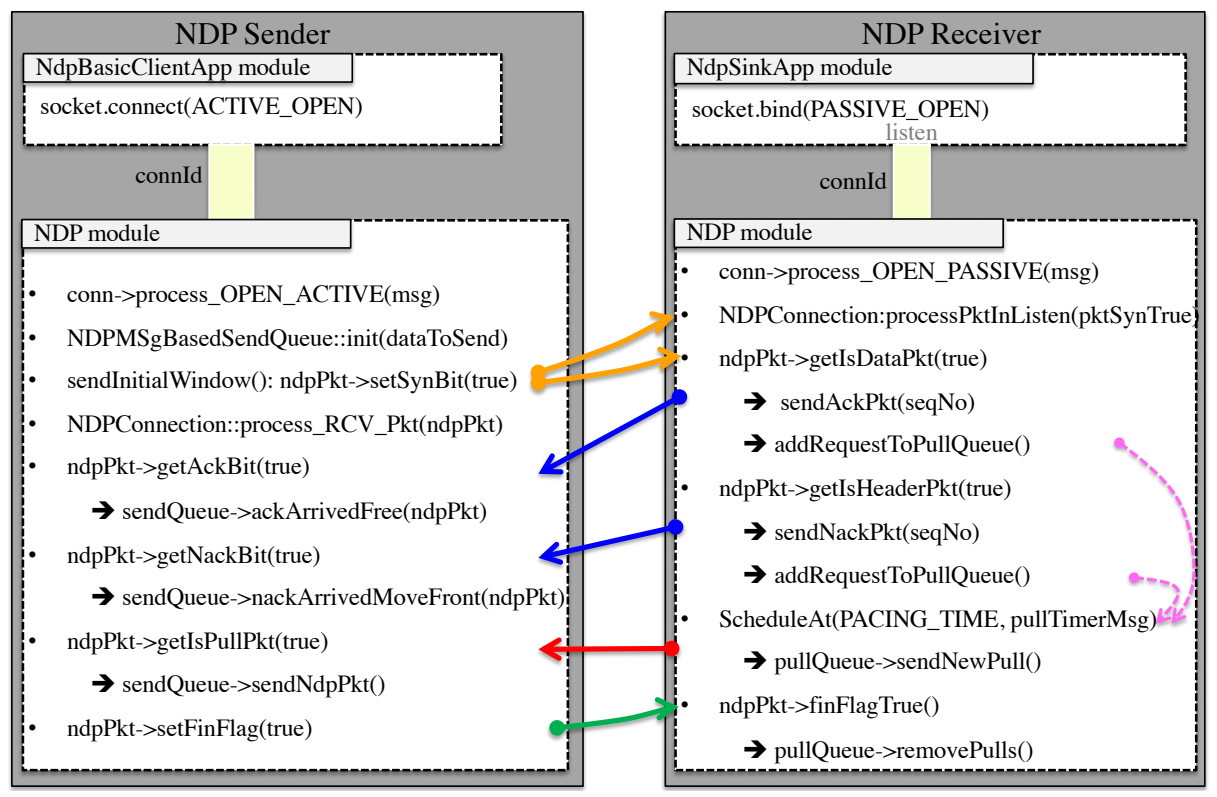

Figure 5: NDP implementation

$N D P_{-} I_{-} D A T A$ : data packet received and $N D P_{-} I_{-} P E E R_{-} C L O S E D$ : FIN flag received from remote NDP. The NDP module and the NDP applications implement NDP operations (as discussed in $\S 4)$ as follows:

1. The NDP sender performs the following operations (see Figure 5):

- creates the sendQueue for the data to be sent: NDPMSgBasedSendQueue::init(data),

- sends the initial window of data packets with SYN flag: sendInitialWindow(),

- precesses received packet (can be ACK, NACK or PULL) from the receiver: NDPConnection::process_RCV_Pkt(ndpPkt). The sender takes one of the following actions:

- frees acknowledged data packet: sendQueue->ackArrivedFree(ndpPkt),

- queues NACKed data packet for retransmission: nackArrivedMoveFront(ndpPkt),

- sends data packet if PULL request arrived: sendQueue->sendNdpPkt(),

- sets the FIN flag when sending the last data packet: ndpPkt->setFinFlag(true).

2. The NDP receiver performs the following operations (see Figure 5):

- establishes a connection when receiving a data packet with the SYN flag set: NDPConnection:processPktInListen(pktSynTrue),

- acknowledges a received data packet: sendAckPkt(seqNo),

- sends NACK when receiving header packet: sendAckPkt(seqNo),

- adds PULL packet to the PULL queue upon receiving either data or header packet: addRequestToPullQueue(),

- schedules a self message that is used to pace pull packets from the PULL queue: ScheduleAt(PACING_TIME, pullTimerMsg)

- removes all pull packets when receiving the last data packet: pullQueue->removePulls()

Network layer. The IPv4 module performs IP encapsulation/decapsulation and routing of datagrams. This is based on function call interface of the IPv4RoutingTable which we updated 
as discussed in $\S 3$.

NICs. We use the available NIC modules (PPPInterface and EthernetInterface) that are provided in the INET Framework.

NDP Switch. It contains two queues: (1) highPriorityQueue: which is used to enqueue Header, ACK, NACK and PULL packets, and (2) dataQueue: which is used to enqueue data packets. NDP switches trim packets when their dataQueue is full and insert the headers in the highPriorityQueue. NDPSwitch:dequeue() is round robin (10:1 ratio) between the two queues.

\section{Experimenting with the Simulation Framework}

We developed a central scheduler node (centralSchedulerNdp ${ }^{3}$ ) to schedule NDP connections in a FatTree topology (as shown in Figure 1). The main parameters in this node are shown in Figure $1 \mathrm{~b}$ and explained below:

- FatTreeSize. This is used to generate a $k$-ary FatTree topology (as described in $\S 2$ )

- trafficMatrixType. The scheduler node can schedule either a permutation or random traffic matrix (as explained in[18])

- arrivalRate. This is used to generate flow arrival times according to a Poisson process. This is implemented by creating an exponential distribution $($ mean $=1 / \lambda)$ based on the Poisson arrival rate $\lambda$.

- flowSize. This is the size of the data (number of packets) that each created NdpBasicClientApp is going to send to an NdpSinkApp.

- numShortFlows. This is the number of NDP connections that will be scheduled based on the selected traffic matrix, e.g. scheduling 1000 flows means that there are 1000 different NdpBasicClientApps communicate with 1000 NdpSinkApps.

- longFlowSize and percentLongFlowNodes. These parameters are used to generate background traffic in the simulation. The background traffic can either run until all short flows will finish or by assigning the size of the long flows to a large flow size. For example, percentLongFlowNodes of $30 \%$ in a 10-FatTree (250 servers) topology means that there are 75 servers that are used only to run background traffic.

- initialWindow and ndpSwitchQueueLength. These are used as described in Section refndpsection).

\subsection{NDP Experiments}

Here, we present experimentation using the developed NDP model. We conducted four different experiments ${ }^{4}$.

Experiment 1. We ran this experiment with the parameters that are shown in Figure $1 \mathrm{~b}$ in a 6FatTree ( 54 servers) where $15 \%$ of servers ( 8 servers) ran background load. The number of short flows were 2000 and each flow had a size of $150 \mathrm{~KB}$. The results of this experiment are shown in Figure 6 (one seed was used). The developed model provides the ability to record and plot the following measurements: goodput (Figure 6a), flow completion time (FCT) (Figure 6b), the number of received header packets per flow (connection) (Figure 6c), the number of data sending applications at each server (Figure 6d), the number of data receiving applications at each server (Figure 6d) and traffic matrix plot (Figure 6e). We use OMNeT++'s scavetool command-line

\footnotetext{
${ }^{3}$ We have developed another scheduler: centralSchedulerTcp, which is used for TCP experiments and it has a similar interface as the NDP scheduler. It is used to schedule TCP connections in a FatTree topology.

${ }^{4}$ Due to space limitations we will not discuss simulations with TCP. Some of these results can be found in [3].
} 


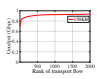

(a) Goodput

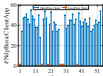

(d) \#created sender's app.

(e) \#created receiver's app.

(f) Perm. traffic matrix

Figure 6: The results of running 2000 NDP short flows (150KB each) in a 6-FatTree (54 servers)

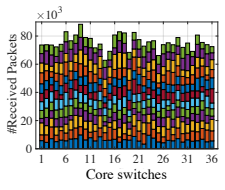

(a) Per-flow ECMP

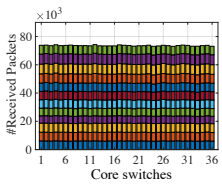

(b) Per-packet ECMP

Figure 7: Per-flow vs per-packet ECMP in 12-FatTree (36 core switches) topology. Each colour in each bar (12 colours) represents the number of received packets at each port (12-port switch)

tool to process the results. These scripts can be found in the shell file (runNdpSimulation.sh) that we use to run the simulation and generate several .csv files which are input to MATLAB scripts to produce the required figures ${ }^{5}$. The results are as expected; most flows achievedvery high goodput and low Flow Completion Times.

Experiment 2. In this experiment, we compare between per-flow and per-packet ECMP in a FatTree topology. We have simulated the two protocols in a 12-FatTree (36 core switches) by running 5000 short flows (300KB each) and with arrival rate $\lambda=2000$ (without introducing any background load). The other parameters are as depicted in Figure 1b. Figure 7 illustrates the number of received packets at each port of the 36 12-port core switches. It is obvious that per-packet ECMP provides better load balancing than per-flow ECMP (as discussed in §3). Experiment 3. In this experiment we test the performance of NDP when varying specific parameters. A key parameter of NDP is the initial window of packets that a sender pushes to the network. In Figure 8a, we observe that for very small values of the initial window, the goodput is very low and the receiver's downlink underutilised. As the window increases, utilisation approaches the maximum available link capacity (for 16 packets). For larger values, the measured goodput is the same (full link capacity). In Figure 8b, we show the goodput of NDP flows at different flow sizes (when $\lambda=2500$ ). Similarly, in Figure 8b, we obtain the goodput of 10000 flows (1.5MB each) at different $\lambda$ values.

Experiment 4. In the experiment, we use realistic web search workloads reported by data centre operators to evaluate NDP [6]. We simulate two target loads of background traffic $(0.5$ and 0.8). We generate 20000 flows, with $\lambda=2500$. We report the flow completion time (FCT) of all flows (split in two different ranges), as shown in Figure 9. NDP achieves low FCT.

\section{Summary and future work}

The proposed model is intended to be used to evaluate NDP and compare its performance with existing models (e.g. TCP) in a FatTree data centre topology. The current implementa-

\footnotetext{
${ }^{5}$ The shell and MATLAB scripts are available in the GitHub repository, along with a demo.
} 


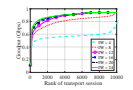

(a) Varying initial window

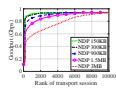

(b) Varying flow size

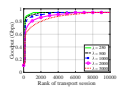

(c) Varying arrival rate $\lambda$

Figure 8: NDP Goodput for 10000 flows in a 10-FatTree when varying specific parameter

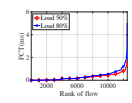

(a) Flow size $(0,100 \mathrm{~KB}]$

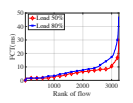

(b) Flow size (100KB, 1MB]

Figure 9: FCT of web search workload flows (two ranges) at different background loads

tion supports most of NDP's specifications. The current version of our NDP model does not support priority pulling at the pull queue. As a future work, we plan to improve the NDP model by allowing priority pacing in the pull queue for short flows. In addition, we aim at leveraging multiple priority queues available in commodity switches to implement a Multiple Level Feedback Queue (MLFQ). Also, we aim at evaluating NDP in Incast and Outcast scenarios and assess the provided fairness among competing flows. Furthermore, we plan to build more OMNeT++/INET-based models that simulate other modern data transport protocols for DCNs, such as DCTCP[6], MPTCP[24], DCQCN[28] and PIAS[8].

\section{References}

[1] Mohammad Al-Fares, Alexander Loukissas, and Amin Vahdat. A scalable, commodity data center network architecture. In Proc. of SIGCOMM, 2008.

[2] Mohammad Al-Fares, Sivasankar Radhakrishnan, Barath Raghavan, Nelson Huang, and Amin Vahdat. Hedera: Dynamic Flow Scheduling for Data Center Networks. In Proc. of USENIX, 2010 .

[3] Mohammed Alasmar, George Parisis, and Jon Crowcroft. Polyraptor: Embracing path and data redundancy in data centres for efficient data transport. In Proceedings of the ACM SIGCOMM 2018 Conference on Posters and Demos, 2018.

[4] Mohammed Alasmar, George Parisis, and Jon Crowcroft. SCDP: Systematic Rateless Coding for Efficient Data Transport in Data Centres. In http://arxiv.org/abs/1909.08928, 2019.

[5] M. Alizadeh, B. Atikoglu, A. Kabbani, A. Lakshmikantha, R. Pan, B. Prabhakar, and M. Seaman. Data center transport mechanisms: Congestion control theory and ieee standardization. In Annual Allerton Conference on Communication, Control, and Computing, 2008.

[6] M Alizadeh, Albert Greenberg, David A Maltz, Jitendra Padhye, Parveen Patel, Balaji Prabhakar, Sudipta Sengupta, and Murari Sridharan. Data Center TCP (DCTCP). In SIGCOMM, 2010.

[7] Mohammad Alizadeh, S Yang, Milad Sharif, Sachin Katti, Nick McKeown, Balaji Prabhakar, and Scott Shenker. pfabric: Minimal near-optimal datacenter transport. In Proc. of SIGCOMM, 2013.

[8] Wei Bai, Li Chen, Kai Chen, Dongsu Han, Chen Tian, and Hao Wang. Information-agnostic flow scheduling for commodity data centers. In In Proc. NSDI, USENIX, 2015.

[9] Pat Bosshart, Dan Daly, Glen Gibb, Martin Izzard, Nick McKeown, Jennifer Rexford, Cole Schlesinger, Dan Talayco, Amin Vahdat, George Varghese, and David Walker. P4: Programming protocol-independent packet processors. In ACM SIGCOMM, 2014.

[10] Yanpei Chen, Rean Griffith, David Zats, Anthony D. Joseph, and Randy Katz. Understanding TCP incast and its implications for big data workloads. In Proc. of USENIX, 2012. 
[11] T. Das and K. M. Sivalingam. Tcp improvements for data center networks. In 2013 Fifth International Conference on Communication Systems and Networks (COMSNETS), 2013.

[12] A Dixit, P Prakash, Y C Hu, and R R Kompella. On the impact of packet spraying in data center networks. In Proc. of INFOCOM, 2013.

[13] Chuanxiong Guo, Haitao Wu, Zhong Deng, Gaurav Soni, Jianxi Ye, Jitu Padhye, and Marina Lipshteyn. Rdma over commodity ethernet at scale. In SIGCOMM, 2016.

[14] Mark Handley, Costin Raiciu, Alexandru Agache, Andrei Voinescu, Andrew W. Moore, Gianni Antichi, and Marcin Wójcik. Re-architecting datacenter networks and stacks for low latency and high performance. In Proc. of SIGCOMM, 2017.

[15] C. Hopps. Analysis of an Equal-Cost Multi-Path Algorithm Status. IETF, RFC 2992, 2000.

[16] Teerawat Issariyakul and Ekram Hossain. Introduction to Network Simulator NS2. Springer Publishing Company, Incorporated, 2010.

[17] M. Kheirkhah, I. Wakeman, and G. Parisis. MMPTCP: A multipath transport protocol for data centers. In Proc. of INFOCOM 2016, 2016.

[18] Morteza Kheirkhah. Mmptcp: a novel transport protocol for data centre networks[. In University of Sussex, 2016.

[19] Morteza Kheirkhah, Ian Wakeman, and George Parisis. Multipath-TCP in ns-3. In WNS3, 2014.

[20] A. R. A. Kumar, S. V. Rao, and D. Goswami. Ns3 simulator for a study of data center networks. In IEEE International Symposium on Parallel and Distributed Computing, 2013.

[21] Behnam Montazeri, Yilong Li, Mohammad Alizadeh, and John Ousterhout. Homa: A receiverdriven low-latency transport protocol using network priorities. In In Proc. ACM SIGCOMM, 2018.

[22] A. Munir, I. A. Qazi, Z. A. Uzmi, A. Mushtaq, S. N. Ismail, M. S. Iqbal, and B. Khan. Minimizing flow completion times in data centers. In IEEE INFOCOM, 2013.

[23] Costin Raiciu, Sebastien Barre, Chris Pluntke, Adam Green, Damon Wischik, and Mark Handley. Improving Datacenter Performance and Robustness with Multipath TCP. In SIGCOMM, 2011.

[24] Costin Raiciu, Christopher Pluntke, Sebastien Barre, Adam Greenhalgh, Damon Wischik, and Mark Handley. Data Center Networking with Multipath TCP. In Proc. of SIGCOMM. ACM, 2010.

[25] Bilel Ben Romdhanne. Large-scale network simulation over heterogeneous computing architecture. In Telecom ParisTech, 2013.

[26] A. Varga. Omnet++ discrete event simulation. In System User Manual, 2006.

[27] H. Xu and B. Li. Repflow: Minimizing flow completion times with replicated flows in data centers. In IEEE INFOCOM, 2014.

[28] Y Zhu, H Eran, Dan F, Chuanxiong G, Marina L, Ye Liron, J Padhye, Shachar R, Mohamad Haj Y, and Ming Zhang. Congestion control for large-scale rdma deployments. In SIGCOMM, 2015. 